\title{
Unusual Metal to Marginal-Metal Transition in Two-Dimensional Ferromagnetic Electron Gases
}

\author{
Weiwei Chen, ${ }^{1}$ C. Wang, ${ }^{2,},{ }^{+}$Qinwei Shi, ${ }^{1}$ Qunxiang Li, ${ }^{1}$ and X. R. Wang ${ }^{3,4,{ }^{\prime}}$ \\ ${ }^{1}$ Hefei National Laboratory for Physical Sciences at the Microscale \& \\ Synergetic Innovation Center of Quantum Information and Quantum Physics, \\ University of Science and Technology of China, Hefei, Anhui 230026, China \\ ${ }^{2}$ School of Electronic Science and Engineering and State Key Laboratory of Electronic Thin Films and Integrated Devices, \\ University of Electronic Science and Technology of China, Chengdu 610054, China \\ ${ }^{3}$ Department of Physics, The Hong Kong University of Science and Technology (HKUST), Clear Water Bay, Kowloon, Hong Kong \\ ${ }^{4}$ HKUST Shenzhen Research Institute, Shenzhen 518057, China
}

(Dated: March 2, 2022)

\begin{abstract}
Two-dimensional ferromagnetic electron gases subject to random scalar potentials and Rashba spin-orbit interactions exhibit a striking quantum criticality. As disorder strength $W$ increases, the systems undergo a transition from a normal diffusive metal consisting of extended states to a marginal metal consisting of critical states at a critical disorder $W_{c, 1}$. Further increase of $W$, another transition from the marginal metal to an insulator occurs at $W_{c, 2}$. Through highly accurate numerical procedures based on the recursive Green's function method and the exact diagonalization, we elucidate the nature of the quantum criticality and the properties of the pertinent states. The intrinsic conductances follow an unorthodox single-parameter scaling law: They collapse onto two branches of curves corresponding to diffusive metal phase and insulating phase with correlation lengths diverging exponentially as $\xi \propto \exp \left[\alpha / \sqrt{\left|W-W_{c}\right|}\right]$ near transition points. Finite-size analysis of inverse participation ratios reveals that the states within the critical regime $\left[W_{c, 1}, W_{c, 2}\right]$ are fractals of a universal fractal dimension $D=1.90 \pm 0.02$ while those in metallic (insulating) regime spread over the whole system (localize) with $D=2$ $(D=0)$. A phase diagram in the parameter space illuminates the occurrence and evolution of diffusive metals, marginal metals, and the Anderson insulators.
\end{abstract}

Anderson localization is a long-lasting fundamental concept in condensed matter physics [1-9] and keeps bringing us surprising, especially in its critical dimensionality of two [10[12]. In the early time, the orthodox view is the absence of diffusion of an initially localized wave packet at an arbitrary weak disorder in one- and two-dimensional electron gases (2DEGs) while metallic states and Anderson localization transitions (ALTs) can occur in 3D [13, 14]. Later more careful renormalization group calculations [15] and numerical simulations [16-20], together with experiments [11], show that intrinsic degrees of freedom can alter the results in 2D: Halfinteger spin particle systems can also support ALTs when the spin rotational symmetry is broken through spin-orbit interactions (SOIs), regardless whether the time reversal symmetry is preserved (symplectic class) [15-20] or not (unitary class) [21-23]. The most unquestionable examples would be quantum Hall effects of both non-interacting [24-26] and interacting [27, 28] 2DEGs in strong perpendicular magnetic fields. On the other hand, all states of disordered non-interacting integer-spin particle systems must be localized [29, 30].

However, recent numerical studies [21--23, 31-34] showed that the current understanding of ALTs in non-interacting 2DEGs is far from completed when SOIs are involved. For example, in contrast to the predictions based on the nonlinear $\sigma$ model that claims only localized states are allowed in the unitary ensemble [15], a band of extended states together with an ALT or critical states usually accompanied by a KosterlitzThouless (KT) transition could exist in 2DEGs without timereversal symmetry, depending on the form of SOIs and the strength of magnetic field [21-23]. In this work, we observe an anomalous phase transition from a normal metal to a marginal metal [35], consisting of a band of metallic critical states, in a ferromagnetic 2DEG on a square lattice subject to a Rashba SOI and random on-site potentials. The statements are supported by argumentations based on two independent highly-accurate numerical approaches: the finite-size scaling analysis of two-terminal conductances and the inverse participation ratios (IPRs) analysis of wave functions obtained from the exact diagonalization. The unaccustomed marginal metal (MM) phase exists between a diffusive metal (DM) phase at weak disorders and an Anderson insulator (AI) phase at strong disorders. Scaling analyses of IPRs show that wave functions of states in the MMs are of fractals of dimension $D=1.90 \pm 0.02$, a feature reminiscent of a band of critical states in the random SU(2) model subject to strong magnetic fields [21].

Our main result is the new (marginal metallic) phase whose $\beta$-function (symbols and black line) defined as $\beta\left(\ln g_{L}\right)=$ $d \ln g_{L} / d \ln L$ describes an unconventional DM-MM-AI transition, as summarized in Fig. 1. In comparison, $\beta$ for other types of phase transitions in $2 \mathrm{D}$ are also included (elucidate in the caption). Here $g_{L}$ and $L$ are dimensionless conductance and system size, respectively. For a large (small) conductance, i.e., $g_{L}>g_{c, 1}\left(g_{L}<g_{c, 2}\right), \beta$ is positive (negative), indicating a metallic (insulating) phase. While between two critical conductances $g_{c, 1}$ and $g_{c, 2}, \beta=0$ shows a MM phase in which conductances are through critical states that are size independent. Different from an ALT at a fixed point $g_{c}[16-20,22,23]$, the MM phase between $\left[g_{c, 1}, g_{c, 2}\right]$ is a fixed line in which the system does not flow away when its size is scaled. Furthermore, near both DM-MM and MM-AI transition points, correlation lengths $\xi$ locating on the DM and AI sides, respectively, diverge with disorder strength $W$ as $\xi(W) \propto \exp \left[\alpha / \sqrt{\left|W-W_{c}\right|}\right]$, a similar finite-size scaling law 


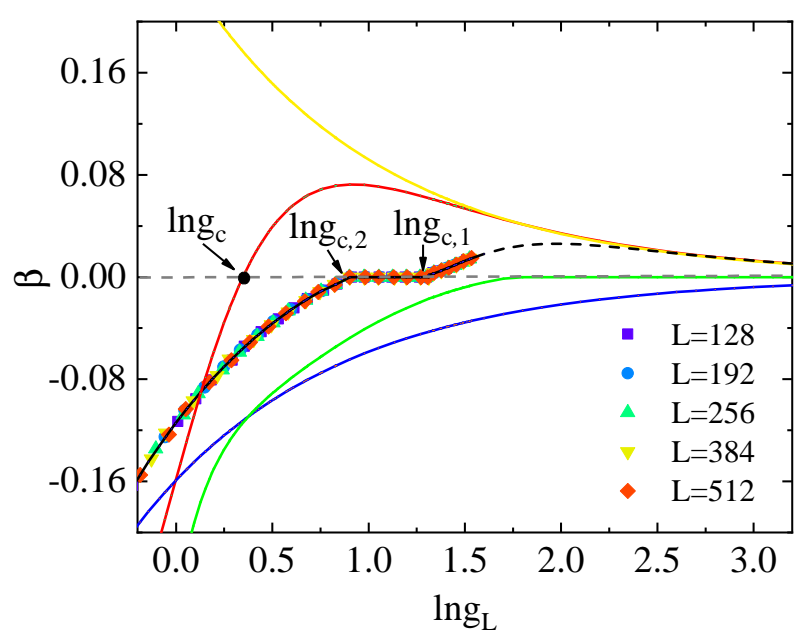

FIG. 1. $\beta\left(\ln g_{L}\right)$ of various $2 \mathrm{D}$ materials. Black solid line stands for the numerical results (symbols in Fig. 22 a) for different system sizes) presented in this work, which displays the coexistence of DM $(\beta>0)$, MM $(\beta=0)$, and AI $(\beta<0)$. While, black dash line is plotted according to an analytical formula [15, 36]. For non-interacting Schrödinger electrons, no delocalization-localization transition is allowed for orthogonal class (blue line) [11, 12], where time-reversal and spin-rotation symmetries are preserved. For symplectic class [16-20, 37], there is one $\beta=0$ point (red line), corresponding to an unstable fixed point in renormalization group flow. The KT transition (green line) from a band of critical states to localized states can also exist [21]. While for the Dirac Hamiltonians, numerical simulations suggest all states are extended in single-valley graphenes [38] (yellow line) while KT transitions are also allowed if random fluxes [31] or intervalley scatterings [35, 39] exist.

in KT transitions (green line) [21, 31, 35].

Our model is a tight-binding Hamiltonian on a square lattice of size $L^{2}$ with unit lattice constant,

$$
H=\sum_{i} c_{i}^{\dagger} \epsilon_{i} c_{i}-\sum_{\langle i, j\rangle} c_{i}^{\dagger} R_{i j} c_{j}
$$

where $c_{i}^{\dagger}=\left(c_{i, \uparrow}^{\dagger}, c_{i, \downarrow}^{\dagger}\right)$ and $c_{i}$ are, respectively, the single electron creation and annihilation operators on site $i=\left(x_{i}, y_{i}\right)$ with $x_{i}, y_{i}$ being integers and $1 \leq x_{i}, y_{i} \leq L$. $\langle i j\rangle$ denotes $i$ and $j$ as the nearest-neighbor sites. The first term stands for on-site energy:

$$
\epsilon_{i}=\epsilon_{0} \sigma_{0}-\Delta \sigma_{z}+V_{i} \sigma_{0}
$$

Here $\sigma_{0}$ and $\sigma_{x, y, z}$ are the 2-by-2 identity and the Pauli matrices respectively. $\epsilon_{0}$ is a constant energy term. $-\Delta \sigma_{z}$ introduces a ferromagnetic term that breaks the time-reversal symmetry with $\Delta$ quantifying the mean-field exchange splitting [40]. Disorders are modelled by $V_{i} \sigma_{0}$ with uncorrelated random numbers $V_{i}$ following the normal distribution of zero mean and the variance of $W^{2}$. Thus, $W$ measures the degree of randomness. A Rashba SOI is encoded in the hopping matrices $R_{i j}$, parameterized by matrices $R_{x}$ and $R_{y}$ along the $x-$ and $y$-directions, respectively,

$$
R_{x}=\frac{1}{2}\left(t \sigma_{0}+i \tilde{\alpha} \sigma_{y}\right) \quad \text { and } \quad R_{y}=\frac{1}{2}\left(t \sigma_{0}-i \tilde{\alpha} \sigma_{x}\right) .
$$

where $t$ is the energy unit and $\tilde{\alpha}$ measures the strength of SOIs. In the clean limit, model (1) can be blocked diagonalized in the momentum space as $H=\sum_{\boldsymbol{k}} c_{\boldsymbol{k}}^{\dagger} h(\boldsymbol{k}) c_{\boldsymbol{k}}$ with

$$
\begin{aligned}
h(\boldsymbol{k})= & \left(\epsilon_{0}-\cos k_{x}-\cos k_{y}\right) \sigma_{0}-\Delta \sigma_{z} \\
& +\tilde{\alpha}\left(\sin k_{x} \sigma_{y}-\sin k_{y} \sigma_{x}\right) .
\end{aligned}
$$

Hereafter we fix $\epsilon_{0}=2$ such that the effective $\boldsymbol{k} \cdot \boldsymbol{p}$ Hamiltonian near the band edge reads $\frac{p^{2}}{2}+\tilde{\alpha}(\boldsymbol{p} \times \boldsymbol{\sigma}) \cdot \hat{z}$. This form of Hamiltonians has been widely employed to enlighten the intrinsic and extrinsic mechanism of the anomalous Hall effect, and possible physical realizations of model (1) include a large family of ferromagnetic semiconductors such as GaAs and other III-V host materials [40].

To investigate the localization properties of states of model (11, we employ the Landauer formula to calculate the dimensionless conductance of a disordered sample between two clean semi-infinite leads at a given Fermi level $E, \tilde{g}_{L}=$ $\operatorname{Tr}\left[T T^{\dagger}\right]$, where $T$ is the transmission matrix [41]. To exclude the contribution from contact resistances, we define the dimensionless conductance $g_{L}$ as, $1 / g_{L}=1 / \tilde{g}_{L}-1 / N_{c}$, where $N_{c}$ is the number of propagating modes in the leads at Fermi energy $E$ [42]. The determination of quantum phase transitions is based on the following criteria: (1) For the Fermi level in the DM (AI) phase, $d g_{L} / d L$ is positive (negative), while in the MM phase, $g_{L}$ is independent of $L$. (2) In the vicinity of phase transition points, there exists the one-parameter scaling hypothesis [13] such that $g_{L}$ of different system sizes merge into a universal smooth scaling function $f(x)$, i.e.,

$$
g_{L}=f(L / \xi)
$$

with the correlation length $\xi$ diverging at the transition points.
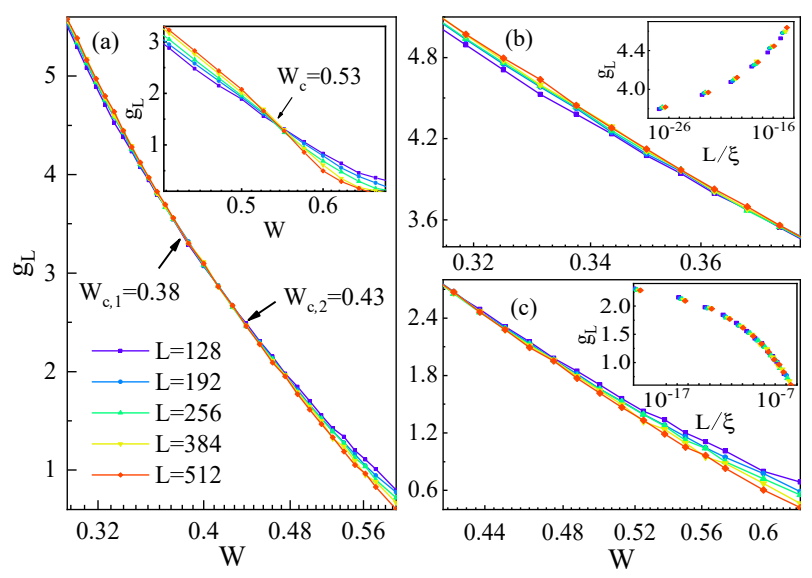

FIG. 2. (a) $g_{L}$ as a function of $W$ for $L=128,192,256,384$, and 512 at $E=0.2$ and $\Delta=0.01$. Inset is the same plot but for $\Delta=0$. (b) Enlargement of the regime near $W_{c, 1}$. Inset: Scaling function $f(x=L / \xi)$ obtained by collapsing data for $W<W_{c, 1}$ into a single curve. (c) Same as (b) but for the regime near $W_{c, 2}$.

Two typical examples are shown in Fig. 2(a) and its inset that plot $g_{L}$ as a function of $W$ for $E=0.2$ (near band edge), $\tilde{\alpha}=0.2$, and $\Delta=0.01$ and 0 (inset), respectively. Clearly, 
in the absence of the ferromagnetic coupling $\Delta=0$ when model (1) belongs to the symplectic class, all curves cross at a single point $W_{c} . d g_{L} / d L$ is positive (negative) for $W$ smaller (larger) $W_{c}$. These features are concrete evidence of an ALT at $W_{c}$. Finite-size scaling analysis [43] shows that $g_{L}$ for different sizes $L$ collapse to a single smooth scaling curve, and $\xi$ diverges as $\left|E-E_{c}\right|^{-v}$ with $v=2.8 \pm 0.2$, consistent with previous estimates [18-20].

Strikingly, once systems enter the unitary class by turning on the ferromagnetic coupling, say $\Delta=0.01$, we observe a MM phase in the window of $W \in\left[W_{c, 1}, W_{c, 2}\right]\left(W_{c, 1}=\right.$ $0.38 \pm 0.01$ and $\left.W_{c, 2}=0.43 \pm 0.01\right)$ within which $d g_{L} / d L=0$ for all $L$, while states for $W<W_{c, 1}$ and $W>W_{c, 2}$ are extended and localized, respectively. Two phase transitions are evident in Figs. 2 (b) and (c), which illustrate the enlargements near $W_{c, 1}$ and $W_{c, 2}$, respectively. The MM-AI transition at $W_{c, 2}$, in addition to the zero-plateau of $\beta$ function shown in Fig. 1. is highly evocative of the KT criticality arising in another unitary ensemble with random SOIs [21] and the graphene with random fluxes or long-range impurities [31, 35]. Nonetheless, the DM-MM transition at $W_{c, 1}$ from a band of extended states to a band of critical states is highly nontrivial since both of them are of metallic phases in the sense that their wave functions spread over the whole lattice (illustrate later). To the best of our knowledge, this kind of disorder-driven metal-metal transitions has never been observed before in 2D materials, but in 3D semimetals [44-48].

To substantiate the validity of one-parameter scaling hypothesis, we show that all curves in Figs. 2 2 b,c) collapse into two smooth functions $f(x=L / \xi)$ shown in the insets of the figures [43], which offer direct verifications of quantum phase transitions at $W_{c, 1}$ and $W_{c, 2}$. On the insulating side and near the phase transition point $W_{c, 2}$, the correlation length is expected to diverge as $\xi \propto \exp \left[\alpha_{2} / \sqrt{\left|W-W_{c, 2}\right|}\right]$ with $\alpha_{2}=8.0 \pm 0.8$, a fingerprint of the KT transitions [21, 31, 35]. Differently, there are no reliable analytical and numerical estimates for the divergence law near the DM-MM transition $W_{c, 1}$. Scaling analysis also suggests $\xi \propto \exp \left[\alpha_{1} / \sqrt{\left|W-W_{c, 1}\right|}\right]$ with the exponent $\alpha_{1}=9 \pm 3$. Besides, a power-law divergence of correlation lengths for ALTs, i.e., $\xi \propto\left|W-W_{c}\right|^{-v}$, also fit the numerical data with $v=32$. However, the obtained $v$ is much larger than any known critical exponents in disordered 2D systems [11, 12]. We thus argue that at the DM-MM transition the correlation lengths show the same scaling behavior as those for KT transitions, rather than the power-law divergence for ALTs.

So far, we have provided one example of the DM-MM-AI transition in model (1). Needless to say, many questions arise, and, among them, the most important one may be the proof of the universality of such a quantum phase transition. In Supplemental Materials [43], we show indications of universality by substantiating emergences of the three phases at different Fermi energies with the same divergence law of $\xi$, i.e., $\xi \propto \exp \left[\alpha / \sqrt{\left|W-W_{c}\right|}\right]$ at critical points. The same physics is observed if we choose $E$ as the scaling variable at a fixed disorder. Furthermore, simulations for a different form of dis- orders and a distinct SOI due to the Dresselhaus effect are both in qualitative agreement with Fig. 2. All these features indicate that the MM phase prevails in ferromagnetic 2DEGs with SOIs and favors the exponential divergence of $\xi$ at critical points.

Having established the universality of DM-MM-AI transitions, we further consider the nature of wave functions in three phases, especially the fractal structure of wave functions in MM phase. Wave functions at an isolated critical point of an ALT or in the critical band are known to have multifractal structures characterizing by a set of anomalous dimensions measuring how their moments scale with sizes [12, 21, 35]. Among them, the fractal dimension plays a pre-eminent role, which is related to the IPR defined as

$$
p_{2}(E, W)=\sum_{i}\left|\psi_{i}(E, W)\right|^{4}
$$

with $\psi_{i}(E, W)$ being the renormalized wave functions of energy $E$ and disorder $W$ at site $i$ for a specific realization. For large enough systems, the average IPR scales with size $L$ as [49-51]

$$
p_{2}(E, W) \propto L^{-D}
$$

with $D$ being the fractal dimension. If the state is extended (localized), $D=d(D=0)$, where $d=2$ is the spatial dimension. While for a critical state, an anomalous scaling with $L$ is expected, i.e., $D \in[0, d]$. Thus, we expect that states in the MM phase have a universal fractal dimension such that their wave functions occupy much sparse space like fractals.
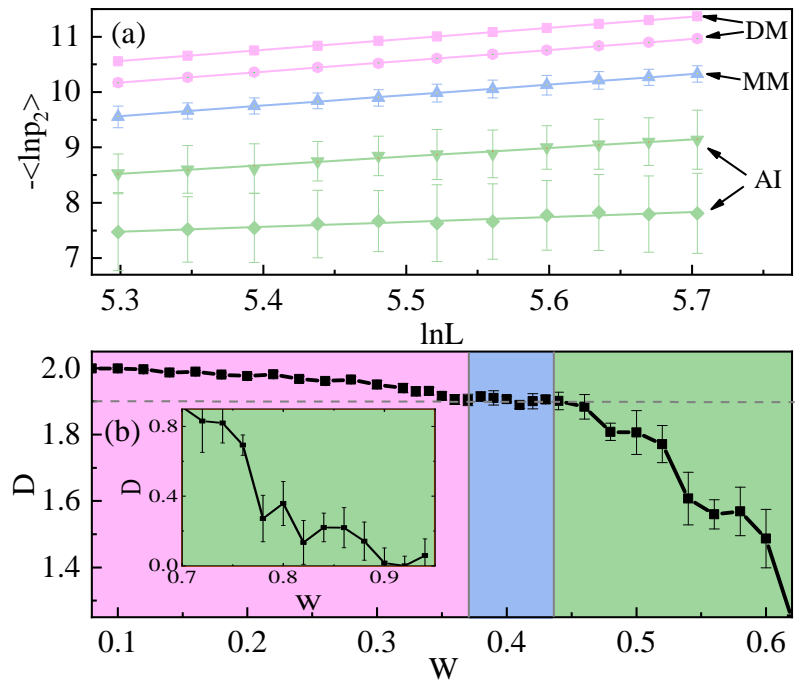

FIG. 3. (a) $-\left\langle\ln p_{2}\right\rangle$ vs $\ln L$ for $E=0.2$. Several disorders in different phases are chosen. DM: $W=0.08$ (squares) and 0.28 (circles); MM: $W=0.42$ (up-triangles); AI: $W=0.56$ (down-triangles) and 0.66 (diamonds). Solid lines are linear fits of numerical data. (b) $D$ as a function of $W$ for $E=0.2$ (squares). Dash line shows the plateau of $D=1.90$. The three phases, colored by magenta (DM), blue (MM), and green (AI), are identified according to data in Fig. 2

Numerically, we use the exact diagonalization to find the eigenfunctions of model (1). In our scenario, we construct 
the tight-binding Hamiltonian by the Kwant package [52] in Python and solve the eigenequation $H \psi=E \psi$ by the Scipy library [53] for $L$ varying from 200 to 300 . The average logarithms of IPR as a function of $\ln L$ for $W=0.08,0.28$, $0.42,0.56$, and 0.66 at $E=0.2$ (the same as Fig. 2(a)) are shown in Fig. 3(a). The corresponding curves are virtually straight lines, which provide strong evidence for the scaling law Eq. (7). The slopes (fractal dimensions) clearly decrease with the increasing of $W$ from the DM phase to the AI phase, and $D=1.90 \pm 0.02$ for $W_{c, 1}<W=0.42<W_{c, 2}$ in the MM phase.

We further authenticate the universality of the fractal nature by displaying $D(W)$ at $E=0.2$ for a large range of disorders covering the three phases in Fig. 3.(b). Apparently, a plateau of $D=1.90$ is observed in the MM phase determined by data in Fig. 2(a), indicating that the fractal dimension of the fixed line does not depend on $W$. For $W<W_{c, 1}$ (DM), wave functions are not a fractal any more since $D \simeq d$, while, for $W \gg W_{c, 2}$, IPRs are found to be independent of $L$, i.e., $D=0$, see the inset of Fig. 3. b), a typical feature for AIs. Noticeably, wave functions near MM-AI transitions and on the localized side, for example $W \in[0.44,0.64]>W_{c, 2}$, have fractal structures as well, which can be contributed to the finite-size effect, rather than the criticality, since $D$ will decrease if we evaluate it by using larger system sizes [43].

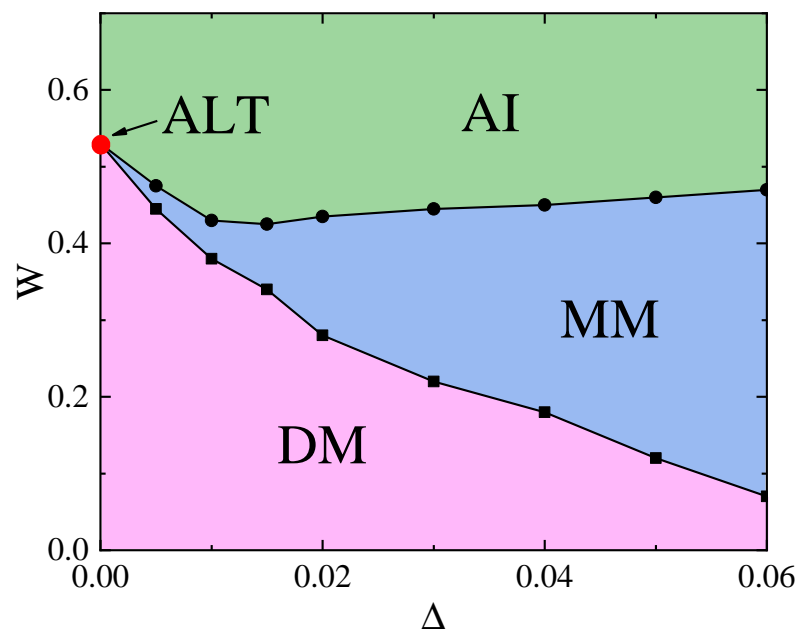

FIG. 4. Schematic phase diagram in the $\Delta-W$ plane: DM (magenta), MM (blue), and AI (green) at $E=0.2$. Only an isolated critical level exists at $\Delta=0$ (symplectic ensemble) with $\xi \propto\left|W-W_{c}\right|^{-v}$ and $v=2.8$. For $\Delta \neq 0$, the MM phase exists within a window of $\left[W_{c, 1}(\Delta), W_{c, 2}(\Delta)\right]$, and $\xi$ in the vicinity of $W_{c, 1}$ (black squares) and $W_{c, 2}$ (black circles) diverges exponentially as $\xi \propto \exp \left[\alpha / \sqrt{\left|W-W_{c}\right|}\right]$.

It is also enlightening to compare the fractal dimensions of the MM phase in model (1) with those of critical states in other 2D materials. The fractal dimension of isolated critical levels for ALTs in the symplectic ensemble is found to be $1.66 \pm$ $0.05[54]$ while $D=1.75$ for the quantum Hall type criticality [55]. Thus, wave functions in the MM phase with $D=1.90 \pm$ 0.02 occupy a larger space than those critical states of random
SU(2) model under strong magnetic fields [21].

A more inclusive picture is procured by executing exhaustive simulations for different $\Delta$ at $E=0.2$. The fixed line persists at finite $\Delta$ as expected such that the three phases coexist, and model (1) always experiences the DM-MM-AI phase transitions at $\Delta$-dependent transition points $W_{c, 1}$ and $W_{c, 2}$. While, for $\Delta=0$, there is only one quantum critical state at which the system undergoes a normal ALT. Furthermore, it is numerically justified that the correlation lengths $\xi$ always diverge as $\xi \propto \exp \left[\alpha / \sqrt{\left|W-W_{c}\right|}\right]$ near $W_{c, 1}$ (squares) and $W_{c, 2}$ (circles) in Fig. 4, see clarifications in Supplemental Materials [43].

In conclusion, analyses of the dimensionless conductance and the IPR provide substantial evidence to the existence of an unusual marginal metallic phase between the diffusive metal and the Anderson insulator in ferromagnetic 2DEGs with SOIs. Such systems undergo a DM-MM-AI transition as either disorder strength or Fermi level varies. Near the transition points, the conductance can be described well by the one-parameter scaling hypothesis. The criticality of the DMMM-AI transitions is consistent with universality description of a quantum phase transition in the sense that correlation lengths diverge as an exponential of an inverse square root of $\left|W-W_{c}\right|$ for all critical points. Besides, eigenfunctions in the MM phase are of fractals of dimension $D=1.90$, while extended states in the DM phase spread over the entire lattice. A schematic phase diagram in the $\Delta-W$ plane is presented.

This work is supported by the National Key Research \& Development Program of China (Grants No. 2016YFA0200604), the National Natural Science Foundation of China (Grants No. 11774296, 11704061, 21873088, and 11874337), and Hong Kong RGC (Grants No. 16301518 and 16300117). C.W. is supported by UESTC and the China Postdoctoral Science Foundation (Grants No. 2017M610595 and 2017T100684). C.W. also acknowledges the support from Peng Yan and Xiansi Wang.

* Corresponding author: cwangad@ connect.ust.hk

Corresponding author: phxwan@ust.hk

[1] P. W. Anderson, Absence of Diffusion in Certain Random Lattices, Phys. Rev. 109, 1492 (1958).

[2] S. John, Electromagnetic Absorption in a Disordered Medium near a Photon Mobility Edge, Phys. Rev. Lett. 53, 2169 (1984)

[3] P. Sheng and Z.-Q. Zhang, Scalar-Wave Localization in a TwoComponent Composite, Phys. Rev. Lett. 57, 1879 (1986)

[4] D. S. Wiersma, P. Bartolini, A. Lagendijk, and R. Righini, Localization of light in a disordered medium, Nature 390, 671 (1997)

[5] M. Störzer, P. Gross, C. M. Aegerter, and G. Maret, Observation of the Critical Regime Near Anderson Localization of Light, Phys. Rev. Lett. 96, 063904 (2006)

[6] A. A. Chabanov, M. Stoytchev, A. Z. Genack, Statistical signatures of photon localization, Nature 404, 850 (2000)

[7] H. Hu, A. Strybulevych, J. H. Page, S. E. Skipetrov, and B. A. van Tiggelen, Localization of ultrasound in a three-dimensional elastic network, Nat. Phys. 4, 945 (2008). 
[8] T. Schwartz, G. Bartal, S. Fishman, M. Segev, Transport and Anderson localization in disordered two-dimensional photonic lattices, Nature 446, 52 (2007)

[9] C. Wang, Y. Cao, X. R. Wang, and P. Yan, Interplay of wave localization and turbulence in spin Seebeck effect, Phys. Rev. B 98, 144417 (2018).

[10] P. A. Lee and T. V. Ramakrishnan, Disordered electronic systems, Rev. Mod. Phys. 57, 287 (1985).

[11] B. Kramer and A. MacKinnon, Localization: theory and experiment, Rep. Prog. Phys. 561469 (1993)

[12] F. Evers and A. D. Mirlin, Anderson transitions, Rev. Mod. Phys. 80, 1355 (2008)

[13] E. Abrahams, P. W. Anderson, D. C. Licciardello, and T. V. Ramakrishnan, Scaling Theory of Localization: Absence of Quantum Diffusion in Two Dimensions, Phys. Rev. Lett. 42, 673 (1979)

[14] D. Friedan, Nonlinear Models in $2+\epsilon$ Dimensions, Phys. Rev. Lett. 45, 1057 (1980)

[15] S. Hikami, Localization, Nonlinear $\sigma$ Model and String Theory, Prog. Theor. Phys. Suppt. 107, 213 (1992)

[16] S. N. Evangelou, Anderson Transition, Scaling, and Level Statistics in the Presence of Spin Orbit Coupling, Phys. Rev. Lett. 75, 2550 (1995)

[17] R. Merkt, M. Janssen, and B. Huckestein, Network model for a two-dimensional disordered electron system with spin-orbit scattering, Phys. Rev. B 58, 4394 (1998)

[18] Y. Asada, K. Slevin, and T. Ohtsuki, Anderson Transition in Two-Dimensional Systems with Spin-Orbit Coupling, Phys. Rev. Lett. 89, 256601 (2002).

[19] P. Markoš and L. Schweitzer, Critical regime of twodimensional Ando model: relation between critical conductance and fractal dimension of electronic eigenstates, J. Phys. A 39, 3221 (2006).

[20] G. Orso, Anderson Transition of Cold Atoms with Synthetic Spin-Orbit Coupling in Two-Dimensional Speckle Potentials, Phys. Rev. Lett. 118, 105301 (2017).

[21] C. Wang, Y. Su, Y. Avishai, Y. Meir, and X. R. Wang, Band of Critical States in Anderson Localization in a Strong Magnetic Field with Random Spin-Orbit Scattering, Phys. Rev. Lett. 114, 096803 (2015)

[22] Y. Su, C. Wang, Y. Avishai, Y. Meir, and X. R. Wang, Absence of localization in disordered two-dimensional electron gas at weak magnetic field and strong spin-orbit coupling, Sci Rep 6 33304 (2016)

[23] C. Wang and X. R. Wang, Anderson transition of twodimensional spinful electrons in the Gaussian unitary ensemble, Phys. Rev. B 96, 104204 (2017)

[24] D. J. Thouless, M. Kohmoto, M. P. Nightingale, and M. den Nijs, Quantized Hall Conductance in a Two-Dimensional Periodic Potential, Phys. Rev. Lett. 49, 405 (1982)

[25] A. M. M. Pruisken, Universal Singularities in the Integral Quantum Hall Effect, Phys. Rev. Lett. 61, 1297 (1988)

[26] B. Huckestein, Scaling theory of the integer quantum Hall effect, Rev. Mod. Phys. 67, 357 (1995)

[27] D. C. Tsui, H. L. Stormer, and A. C. Gossard, Two-Dimensional Magnetotransport in the Extreme Quantum Limit, Phys. Rev. Lett. 48, 1559 (1982)

[28] R. B. Laughlin, Anomalous Quantum Hall Effect: An Incompressible Quantum Fluid with Fractionally Charged Excitations, Phys. Rev. Lett. 50, 1395 (1983)

[29] R. Sepehrinia, Universality of Anderson transition in twodimensional systems of symplectic symmetry class, Phys. Rev. B 81, 045104 (2010)

[30] Y. Su and X. R. Wang, Role of spin degrees of freedom in An- derson localization of two-dimensional particle gases with random spin-orbit interactions, Phys. Rev. B 98, 224204 (2018)

[31] X. C. Xie, X. R. Wang, and D. Z. Liu, Kosterlitz-Thouless-Type Metal-Insulator Transition of a 2D Electron Gas in a Random Magnetic Field, Phys. Rev. Lett. 80, 3563 (1998)

[32] G. Xiong, S.-D. Wang, Q. Niu, D.-C. Tian, and X. R. Wang, Metallic Phase in Quantum Hall Systems due to Inter-LandauBand Mixing, Phys. Rev. Lett. 87, 216802 (2001)

[33] Y. Avishai and Y. Meir, New Spin-Orbit-Induced Universality Class in the Integer Quantum Hall Regime, Phys. Rev. Lett. 89, 076602 (2002)

[34] J. Bang and K. J. Chang, Localization and one-parameter scaling in hydrogenated graphene, Phys. Rev. B 81, 193412 (2010)

[35] Y.-Y. Zhang, J. Hu, B. A. Bernevig, X. R. Wang, X. C. Xie, and W. M. Liu, Localization and the Kosterlitz-Thouless Transition in Disordered Graphene, Phys. Rev. Lett. 102, 106401 (2009).

[36] We cannot use the numerical data at hand to depict $\beta\left(g_{L}\right)$ for large $g_{L}$. Instead, at this stage, we use an analytical formula $\beta(g)=f_{1} g^{-1}+f_{4} g^{-4}+o\left(g^{-5}\right)$ based on the non-linear $\sigma$ model [15] with $f_{1}$ and $f_{4}$ being fitting parameters. The exhaustive illustration of $\beta(g)$ in the large $g$ limit surely deserves further efforts, which, however, is not the main focus in this work.

[37] Y. Asada, K. Slevin, and T. Ohtsuki, Numerical estimation of the $\beta$ function in two-dimensional systems with spin-orbit coupling, Phys. Rev. B 70, 035115 (2004)

[38] J. H. Bardarson, J. Tworzydło, P. W. Brouwer, and C. W. J. Beenakker, One-Parameter Scaling at the Dirac Point in Graphene, Phys. Rev. Lett. 99, 106801 (2007)

[39] Z. Xu, L. Sheng, R. Shen, B. Wang, and D. Y. Xing, KosterlitzThouless transition in disordered two-dimensional topological insulators, J. Phys. Condens. Matter 25, 065501 (2013).

[40] N. Nagaosa, J. Sinova, S. Onoda, A. H. MacDonald, and N. P. Ong, Anomalous Hall effect, Rev. Mod. Phys. 82, 1539 (2010)

[41] A. MacKinnon, The calculation of transport properties and density of states of disordered solids, Z. Phys. B 59, 385 (1985)

[42] S. Datta, Electronic Transport in Mesoscopic Systems (Cambridge University Press, Cambridge, England, 1995).

[43] See Supplemental Materials at http://link.aps.org/supplemental

[44] P. Goswami and S. Chakravarty, Quantum criticality between topological and band insulators in $3+1$ dimensions, Phys. Rev. Lett. 107, 196803 (2011)

[45] K. Kobayashi, T. Ohtsuki, K. T. Imura, and I. F. Herbut, Density of states scaling at the semimetal to metal transition in three dimensional topological insulators, Phys. Rev. Lett. 112, 016402 (2014)

[46] C.-Z. Chen, J. Song, H. Jiang, Q. Sun, Z. Wang, and X. C. Xie, Disorder and Metal-Insulator Transitions in Weyl Semimetals, Phys. Rev. Lett. 115, 246603 (2015)

[47] B. Fu, W. Zhu, Q. Shi, Q. Li, J. Yang, and Z. Zhang Accurate Determination of the Quasiparticle and Scaling Properties Surrounding the Quantum Critical Point of Disordered ThreeDimensional Dirac Semimetals, Phys. Rev. Lett. 118, 146401 (2017)

[48] B. Roy and M. S. Foster, Quantum Multicriticality near the Dirac-Semimetal to Band-Insulator Critical Point in Two Dimensions: A Controlled Ascent from One Dimension, Phys. Rev. X 8, 011049 (2018)

[49] X. R. Wang, Y. Shapir, and M. Rubinstein, Analysis of multiscaling structure in diffusion-limited aggregation: A kinetic renormalization-group approach, Phys. Rev. A 39, 5974 (1989)

[50] M. Janssen, Statistics and scaling in disordered mesoscopic electron systems, Phys. Rep. 295, 1 (1998).

[51] J. H. Pixley, P. Goswami, and S. Das Sarma, Anderson Localization and the Quantum Phase Diagram of Three Dimensional 
Disordered Dirac Semimetals, Phys. Rev. Lett. 115, 076601 (2015)

[52] C. W. Groth, M. Wimmer, A. R. Akhmerov, and X. Waintal, Kwant: a software package for quantum transport, New J. Phys. 16, 063065 (2014)

[53] E. Jones, E. Oliphant, P. Peterson, et al., SciPy: Open Source Scientific Tools for Python, [Online; accessed 2019-05-24]

[54] H. Obuse and K. Yakubo, Anomalously localized states and multifractal correlations of critical wave functions in twodimensional electron systems with spin-orbital interactions, Phys. Rev. B 69, 125301 (2004)

[55] F. Evers, A. Mildenberger, and A. D. Mirlin, Multifractality at the spin quantum Hall transition, Phys. Rev. B 67, 041303(R) (2003) 